\title{
Transcriptional regulatory circuits controlling mitochondrial biogenesis and function
}

\author{
Daniel P. Kelly ${ }^{1,3}$ and Richard C. Scarpulla ${ }^{2}$ \\ ${ }^{1}$ Center for Cardiovascular Research, Departments of Medicine, Molecular Biology \& Pharmacology, and Pediatrics, \\ Washington University School of Medicine, St. Louis, Missouri 63119, USA; ${ }^{2}$ Department of Cell and Molecular Biology, \\ Northwestern Medical School, Chicago, Illinois 60611, USA
}

We are witnessing a period of renewed interest in the biology of the mitochondrion. The mitochondrion serves a critical function in the maintenance of cellular energy stores, thermogenesis, and apoptosis. Moreover, alterations in mitochondrial function contribute to several inherited and acquired human diseases and the aging process. This review summarizes our understanding of the transcriptional regulatory mechanisms involved in the biogenesis and energy metabolic function of mitochondria in higher organisms.

\section{The mitochondrial genome}

A defining feature of eukaryotic cells is that they contain nuclear and mitochondrial genomes sequestered into distinct subcellular compartments. The mitochondrial genetic system is comprised of a circular DNA genome (mtDNA, $\sim 16.5 \mathrm{~kb}$ in vertebrates; Fig. 1), the enzymes required for its transcription and replication, and the protein synthetic machinery necessary for the translation of 13 mitochondrial mRNAs (for review, see Garesse and Vallejo 2001). These mRNAs, which account for the entire protein-coding capacity of mtDNA, encode essential subunits of respiratory complexes I, III, IV, and V. The extrusion of protons through complexes I, III, and IV is coupled to the sequential transfer of electrons to a series of carriers of increasing redox potential resulting in an electrochemical proton gradient across the inner membrane. Complex V, comprised of an ATPase coupled to an inner membrane proton channel, can dissipate the proton gradient in the synthesis of ATP or can couple proton pumping to ATP hydrolysis to maintain the gradient. mtDNA also encodes for two ribosomal and 22 transfer RNAs, required for translation by mitoribosomes within the matrix.

\footnotetext{
${ }^{3}$ Corresponding author.
}

E-MAIL dkelly@im.wustl.edu; FAX (314) 362-0186.

Article and publication are at http://www.genesdev.org/cgi/doi/10.1101/ gad.1177604.
The limited coding capacity of mtDNA necessitates that nuclear genes make a major contribution to mitochondrial metabolic systems and molecular architecture (Garesse and Vallejo 2001). One major class of nuclear genes contributes catalytic and auxiliary proteins to the mitochondrial enzyme systems. For example, the majority of the 100 or so subunits of the respiratory apparatus are nucleus-encoded. In addition, nucleus-encoded metabolic enzymes necessary for the oxidation of pyruvate, fatty acids ( $\beta$-oxidation cycle), and acetyl-CoA (tricarboxylic acid cycle), the biosynthesis of certain amino acids, and the manufacture of heme, among others, are localized to the mitochondrion. A second class of nuclear genes encodes protein import and assembly factors. A third class contributes key proteins that are required for the replication and expression of the mitochondrial genome including nucleic acid polymerases, RNA processing enzymes, transcription and replication factors as well as tRNA-synthetases, translation factors, and ribosomal subunits. Thus, the program regulating mitochondrial biogenesis involves the coordinate actions of nuclear and mitochondrial genes.

\section{Regulatory proteins involved in mitochondrial gene transcription}

In yeast, mtDNA transcription is initiated at $\sim 20$ transcriptional units throughout the genome (for review, see Poyton and McEwen 1996). In vertebrates, transcription is initiated bidirectionally at two promoters, $\mathrm{P}_{\mathrm{H}}$ and $\mathrm{P}_{\mathrm{L}}$ for heavy $(\mathrm{H})$ and light strands $(\mathrm{L})$, respectively, within the D-loop regulatory region (Shadel and Clayton 1997; Clayton 2000). The D-loop is the longest noncoding region in vertebrate mtDNA and contains, in addition to $\mathrm{P}_{\mathrm{H}}$ and $\mathrm{P}_{\mathrm{L}}$, the $\mathrm{H}$-strand replication origin $\left(\mathrm{O}_{\mathrm{H}} ;\right.$ Fig. 1). In the "strand asymmetric model" of mtDNA replication, the RNA transcript initiated at $\mathrm{P}_{\mathrm{L}}$ is cleaved in the vicinity of three evolutionarily conserved sequence blocks (CSB I, II, and III), and $\mathrm{H}$-strand replication is initiated at the sites of these cleavages (Bogenhagen and Clayton 2003). Thus, transcription is coupled to DNA replication 
Figure 1. Human mitochondrial DNA (mtDNA). The genomic organization and structural features of human mtDNA are depicted in a circular genomic map. The D-loop regulatory region is expanded and shown above. Protein coding and rRNA genes are interspersed with 22 tRNA genes (denoted by the single-letter amino acid code). The D-loop regulatory region contains the $\mathrm{L}$ - and $\mathrm{H}$-strand promoters $\left(\mathrm{P}_{\mathrm{L}}\right.$ and $\mathrm{P}_{\mathrm{H}}$, respectively) along with the origin of $\mathrm{H}$-strand replication $\left(\mathrm{O}_{\mathrm{H}}\right)$. mtDNA transcription complexes containing mitochondrial RNA polymerase, Tfam, and TFB are depicted in the expanded D-loop along with the conserved sequence blocks (CSB I, II, and III). The origin of L-strand replication $\left(\mathrm{O}_{\mathrm{L}}\right)$ is displaced by approximately two-thirds of the genome within a cluster of five tRNA genes. Protein-coding genes include cytochrome oxidase $(C O X)$ subunits 1,2 , and 3; NADH dehydrogenase (ND) subunits $1,2,3,4$, $4 \mathrm{~L}, 5$, and 6 ; ATP synthase (ATPS) subunits 6 and 8 ; and cytochrome $b(C y t b)$. ND6 and the eight tRNA genes encoded on the L-strand are in bold type and underlined; all other genes are encoded on the H-strand.

and the sites of RNA cleavage are transition sites between RNA and DNA synthesis. A decision must be made to continue transcription through the CSBs or to truncate the nascent RNA to initiate DNA replication. After DNA synthesis begins, the nascent strand is often terminated downstream from a conserved element referred to as a termination-associated sequence (TAS). This event accounts for the triple-stranded D-loop structure and may be important in controlling mtDNA levels (Brown and Clayton 2002).

The mitochondrial $\mathrm{H}$ - and L-strand transcriptional units differ from most nuclear genes in that they are polygenic. In addition to the RNA primer for $\mathrm{H}$-strand replication, $\mathrm{P}_{\mathrm{L}}$ also directs the synthesis of a transcript that is processed to one mRNA and eight of the 22 tRNAs. The polygenic transcript directed by $\mathrm{P}_{\mathrm{H}}$ is processed to 14 tRNAs, 12 mRNAs, and the two rRNAs (for review, see Garesse and Vallejo 2001). Both promoters share a critical upstream enhancer that serves as the recognition site for mitochondrial transcription factor A or Tfam (previously mtTF-1 and mtTFA), an HMG-box protein that stimulates transcription through specific binding to upstream enhancers. Like other HMG proteins, Tfam can bend and unwind DNA, properties potentially linked to its ability to stimulate transcription upon binding DNA (Fisher et al. 1992). In addition to specific promoter recognition, Tfam binds nonspecific DNA with high affinity. This property along with its abundance in mitochondria suggests that it plays a role in the stabilization and maintenance of the mitochondrial chromosome through its phased binding to nonpromoter sites (Parisi et al. 1993).
Several lines of evidence indicate that Tfam is required for mtDNA replication and maintenance. Tfam knockout mice display embryonic lethality and depletion of mtDNA (Larsson et al. 1998). In addition, Tfam levels correlate well with increased mtDNA in ragged-red muscle fibers and decreased mtDNA levels in mtDNAdepleted cells (Larsson et al. 1994; Poulton et al. 1994). ABF2, a related HMG-box yeast factor, is required for mtDNA maintenance and respiratory competence (Diffley and Stillman 1991). Expression of Tfam can complement an ABF2 deficiency in yeast, suggesting that the two proteins are functionally homologous (Parisi et al. 1993). Interestingly, despite this functional complementation, ABF2 lacks an activation domain present in Tfam and does not stimulate transcription (Dairaghi et al. 1995).

Significant progress has been made in the characterization of the mtDNA transcription initiation machinery. A vertebrate mitochondrial RNA polymerase and a specificity factor that are required for mitochondrial-specific initiation were initially identified and characterized in Xenopus laevis (Antoshechkin and Bogenhagen 1995; Bogenhagen 1996). Although purification of the human polymerase has been elusive, a human cDNA that encodes a protein with sequence similarity to yeast mitochondrial and phage polymerases has been identified in database screenings (Tiranti et al. 1997). The encoded protein localizes to mitochondria, suggesting that it is a bona fide mitochondrial polymerase. A human mitochondrial transcription factor B (h-mtTFB) cDNA has also been isolated, and the encoded protein has properties consistent with it being a functional homolog of the 
yeast specificity factor, sc-mtTFB (McCulloch et al. 2002). The protein is localized to mitochondria and can bind DNA and stimulate transcription from an L-strand promoter in vitro. Subsequently, two isoforms of h-mtTFB, termed TFB1 and 2, were identified (Falkenberg et al. 2002). TFB1 is identical to the initial h-mtTFB isolate. Like the yeast factor, both TFBs share sequence similarities with rRNA dimethyltransferases, although the similarity between TFB2 and this class of enzymes is weaker than that of TFB1. Both TFB isoforms can support specific initiation from mitochondrial promoters in an in vitro system containing purified recombinant proteins. In this system, the TFB-dependent activation of transcription depends on mitochondrial RNA polymerase and Tfam (Fig. 1). Both TFBs interact with mitochondrial RNA polymerase, but TFB1 has about one-tenth the transcriptional activity of TFB2. In addition to binding mitochondrial RNA polymerase, TFB1 also contacts the C-terminal domain of Tfam (McCulloch and Shadel 2003). The region of contact between TFB1 and Tfam is essential for transcriptional activation and corresponds to a 29-amino-acid domain that was previously identified as a Tfam activation domain (Dairaghi et al. 1995). This reinforces the distinction between Tfam and the yeast HMG-box protein ABF2, which, like Tfam, is required for mtDNA maintenance but does not function as a transcription factor.

\section{Transcriptional regulators of nuclear encoded mitochondrial proteins: the critical role of nuclear respiratory factors 1 and 2}

The cytochrome $c$ and cytochrome oxidase genes have served as the prototypes for identifying regulatory factors that act on nuclear respiratory genes from both yeast and mammalian cells. Early work in yeast demonstrated that transcriptional regulation of the major cytochrome $c$ isoform, CYC1, was mediated by oxygen and carbon sources through the upstream activation sites, UAS1 and UAS2. This work has been the subject of excellent reviews to which the reader is referred for original citations ( $\mathrm{Zi}$ tomer and Lowry 1992; Poyton and McEwen 1996).

The identification of nucleus-encoded transcription factors required for the expression of the respiratory apparatus in mammalian cells also began with the characterization of the cytochrome $c$ gene (for reviews, see Scarpulla 1997, 1999). Interestingly, the mammalian cytochrome $c$ promoter has multiple recognition sites for transcription factors that bear no obvious relationship to those identified in yeast (Evans and Scarpulla 1988). A potent cis-acting element, localized to the first intron, consists of tandem Sp1 recognition sites that function synergistically to maximize promoter activity. A second cis-element binds transcription factors of the ATF/CREB family (Evans and Scarpulla 1989). The cytochrome c promoter also contains a recognition site for a transcription factor designated nuclear respiratory factor 1, or NRF-1 (Evans and Scarpulla 1989). NRF-1 is a $68-\mathrm{kD}$ polypeptide with the presence of a C-terminal transcriptional activation domain comprised of glutamine-con- taining clusters of hydrophobic amino acid residues (Chau et al. 1992; Gugneja et al. 1996). Both endogenous and recombinant proteins bind as a homodimer to palindromic NRF-1 sites through guanine nucleotide contacts over a single turn of the DNA helix (Virbasius et al. 1993a). Serine phosphorylation of the N-terminal domain of NRF-1 enhances both its DNA-binding (Gugneja and Scarpulla 1997) and trans-activation functions (Herzig et al. 2000).

NRF-1 has been linked to the transcriptional control of many genes involved in mitochondrial function and biogenesis (Table 1). NRF-1 target genes have been identified by characterization of functional NRF-1-binding sites within their promoters. Many NRF-1 target genes encode subunits of the five respiratory complexes (Virbasius et al. 1993a). However, the regulatory network controlled by NRF-1 extends beyond the respiratory subunits to other classes of genes. These include genes involved in assembly of the respiratory apparatus, constituents of the mtDNA transcription and replication machinery, mitochondrial and cytosolic enzymes of the heme biosynthetic pathway, and components of mitochondrial protein import. Notably, Tfam is an NRF-1 target gene consistent with the postulate that NRF-1 plays an integrative role in nucleo-mitochondrial interactions. This hypothesis has been reinforced by the results of several recent studies associating increases in NRF-1 mRNA levels or DNA-binding activity with mitochondrial biogenesis. NRF-1 and its coactivator PGC-1 (see below) are induced as part of the adaptation of skeletal muscle to exercise training (Murakami et al. 1998; Baar et al. 2002). Similar results were obtained in cultured myotubes in response to elevated calcium, which mimics exercise-induced mitochondrial biogenesis (Ojuka et al. 2003). Likewise, treatment of rats with a creatine analog that induces muscle adaptations analogous to those observed during exercise leads to the activation of AMP-activated protein kinase and increased NRF-1-DNA binding activity, cytochrome c content, and mitochondrial density (Bergeron et al. 2001). Both NRF-1 and Tfam mRNAs are elevated in cells depleted of mtDNA, presumably as a response to increased oxidative stress (Miranda et al. 1999|. Lastly, NRF-1 and Tfam are up-regulated in response to lipopolysaccharide-induced oxidative damage to mitochondria, presumably to enhance mtDNA levels and OXPHOS activity (Suliman et al. 2003).

Perhaps the strongest in vivo link between NRF-1 and the control of mitochondrial function comes from the results of targeted disruption of the NRF-1 gene in mice (Huo and Scarpulla 2001). Homozygosity of the null allele results in lethality between embryonic days 3.5 and 6.5 (E3.5 and E6.5). The null blastocysts fail to grow in culture despite having a normal morphology. Homozygous null blastocysts are defective in maintaining a mitochondrial membrane potential and have severely reduced mtDNA levels. This is not accompanied by increased apoptosis, making it unlikely that the reduction in mtDNA is associated with a generalized increase in DNA fragmentation. Moreover, the mature oocytes of 
Table 1. Nuclear and mitochondrial genes with NRF-1 and NRF-2 recognition sites

\begin{tabular}{|c|c|c|}
\hline & NRF-1 ${ }^{\mathrm{a}}$ & NRF-2 ${ }^{\mathrm{a}}$ \\
\hline \multicolumn{3}{|l|}{ Oxidative phosphorylation } \\
\hline Rat cytochrome $c$ & + & \\
\hline Human cytochrome $c$ & + & \\
\hline \multicolumn{3}{|l|}{ Complex I: } \\
\hline Human NADH dehydrogenase subunit & & \\
\hline 8 (TYKY) & + & \\
\hline \multicolumn{3}{|l|}{ Complex II: } \\
\hline $\begin{array}{l}\text { Human succinate dehydrogenase } \\
\text { subunit B }\end{array}$ & + & + \\
\hline $\begin{array}{l}\text { Human succinate dehydrogenase } \\
\text { subunit C }\end{array}$ & + & + \\
\hline $\begin{array}{l}\text { Human succinate dehydrogenase } \\
\text { subunit D }\end{array}$ & + & + \\
\hline \multicolumn{3}{|l|}{ Complex III: } \\
\hline Human ubiquinone-binding protein & + & \\
\hline Human core protein I & + & \\
\hline \multicolumn{3}{|l|}{ Complex IV: } \\
\hline Rat cytochrome oxidase subunit IV & & + \\
\hline Mouse cytochrome oxidase subunit IV & & + \\
\hline Mouse cytochrome oxidase subunit $\mathrm{Vb}$ & + & + \\
\hline Rat cytochrome oxidase subunit $\mathrm{Vb}$ & + & + \\
\hline $\begin{array}{l}\text { Human/primate cytochrome oxidase } \\
\text { subunit } \mathrm{Vb}\end{array}$ & + & + \\
\hline Rat cytochrome oxidase subunit VIc & + & \\
\hline Human cytochrome oxidase subunit & & \\
\hline VIaL & + & + \\
\hline Bovine cytochrome oxidase subunit & & \\
\hline VIIaL & + & + \\
\hline Human cytochrome oxidase subunit & & \\
\hline VIIaL & & + \\
\hline Bovine cytochrome oxidase subunit & & \\
\hline VIIc & & + \\
\hline \multicolumn{3}{|l|}{ Complex V: } \\
\hline Bovine ATP synthase $\gamma$ subunit & + & \\
\hline Human ATP synthase $\mathrm{c}$ subunit & + & \\
\hline Human ATP synthase $\beta$ subunit & & + \\
\hline \multicolumn{3}{|l|}{ mtDNA transcription and replication } \\
\hline Human Tfam & + & + \\
\hline Mouse Tfam & & + \\
\hline Rat Tfam & & + \\
\hline Mouse MRP RNA & + & \\
\hline Human MRP RNA & + & \\
\hline Human TFB1 & + & + \\
\hline Mouse TFB1 & & + \\
\hline Human TFB2 & + & + \\
\hline Mouse TFB2 & & + \\
\hline \multicolumn{3}{|l|}{ HEME biosynthesis } \\
\hline Rat 5 -aminolivulinate synthase & + & \\
\hline Mouse uroporphyrinogen III synthase & + & + \\
\hline \multicolumn{3}{|l|}{ Protein import and assembly } \\
\hline Human Tom 20 & + & + \\
\hline Human Tom 70 & & + \\
\hline Mouse chaperonin 10 & + & \\
\hline Human SURF-1 & & + \\
\hline Mouse COX17 & + & + \\
\hline
\end{tabular}

Table 1. (continued)

\begin{tabular}{lcc}
\hline & NRF-1 $^{\mathrm{a}}$ & NRF-2 $^{\mathrm{a}}$ \\
\hline Ion channels & & \\
Human VDAC3 & + & \\
Mouse VDAC3 & + & + \\
$\quad$ Human VDAC1 & \\
Shuttles & \\
$\quad$ Human glycerol phosphate & + \\
$\quad$ dehydrogenase & & + \\
Translation & & + \\
$\quad$ Human mitochondrial ribosomal S12 & + & + \\
\hline
\end{tabular}

${ }^{\mathrm{a}}$ Original references for the majority of the indicated NRF-1 and/or NRF-2 target genes that are related to mitochondrial function have been cited elsewhere (Scarpulla 1997, 2002). New additions include human Tomm 70 (Blesa et al. 2003) and mouse COX 17 (Takahashi et al. 2002).

heterozygous mothers have a normal complement of mtDNA, supporting the argument against a defect in mtDNA amplification during oogenesis. Therefore, the mtDNA depletion occurs between fertilization and the blastocyst stage and most likely results from the loss of a NRF-1-dependent pathway of mtDNA maintenance. Interestingly, Tfam-null embryos also exhibit severely depleted levels of mtDNA but survive to E8.5-E10.5 (Larsson et al. 1998). Thus, it is likely that the early mortality of NRF-1-null embryos results from the combined effects of reduced levels of mtDNA and disruption of other NRF-1-dependent functions.

Characterization of cytochrome oxidase genes led to the identification of a second regulatory factor designated as NRF-2 (for review, see Scarpulla 1997, 1999). A series of directly repeated NRF-2 sites within the mouse COXIV promoter overlaps multiple transcription initiation sites and contains additional binding sites for the ETS-domain family of transcription factors (Virbasius and Scarpulla 1991; Carter et al. 1992). The complex binding the NRF-2 sites was purified to homogeneity from HeLa cell nuclear extracts and is comprised of five subunits. These include a DNA-binding $\alpha$ subunit and four others $\left(\beta_{1}, \beta_{2}, \gamma_{1}\right.$, and $\left.\gamma_{2}\right)$ that complex with $\alpha$ but alone do not bind DNA. The NRF-2 complexes activate transcription through four directly repeated ETS-domain-binding sites in the $C O X V b$ promoter, suggesting that NRF-2 may also act on multiple respiratory promoters (Virbasius et al. 1993b).

Purification and molecular cloning of all five NRF-2 subunits established that NRF-2 is the human homolog of mouse GABP (LaMarco and McKnight 1989) and that the two additional human subunits, $\beta_{1}$ and $\gamma_{1}$, were minor splice variants of GABP subunits $\beta_{1}$ and $\beta_{2}$ (Gugneja et al. 1995). The function of the non-DNA-binding subunits is twofold. First, the GABP $\beta_{1}$ subunit, corresponding to NRF- $2 \beta_{1}$ and NRF- $2 \beta_{2}$ (Gugneja et al. 1995), has a dimerization domain that facilitates cooperative binding of a heterotetrameric complex to tandem binding sites (Thompson et al. 1991). In solution, GABP exists as 
an $\alpha \beta$ heterodimer but is induced to form the heterotetramer $\alpha_{2} \beta_{2}$ by DNA containing two or more binding sites (Chinenov et al. 2000). The crystal structure of the heterotetramer bound to DNA has been determined (Batchelor et al. 1998). The second function of the nonDNA-binding subunits is to contribute a transcriptional activation domain. This domain resembles that found in NRF-1 and has been localized to a region upstream from the homodimerization domain (Gugneja et al. 1996).

Functional NRF-2 sites have now been identified in several COX promoters as well as in many other genes related to respiratory chain expression (for review, see Scarpulla 2002). As with NRF-1, the list of respiratory genes containing NRF-2 sites has expanded in recent years (Table 1). These include genes for Tfam (Larsson et al. 1998; Rantanen et al. 2001) and the newly discovered TFB factors (Falkenberg et al. 2002; McCulloch et al. 2002) involved in mitochondrial transcription and DNA replication (Rantanen et al. 2001). Genes encoding three of the four human succinate dehydrogenase (complex II) subunits also have both NRF-1 and NRF-2 sites in their promoters (Au and Scheffler 1998; Elbehti-Green et al. 1998; Hirawake et al. 1999). In many cases, NRF-1 sites are also present in NRF-2-dependent promoters, but this is not a general rule. For example, several $C O X$ promoters and the rodent Tfam (Choi et al. 2002) and TFB (Rantanen et al. 2003) promoters do not have obvious NRF-1 consensus sites (Table 1). This contrasts with the human Tfam (Virbasius and Scarpulla 1994) and TFB (R.C. Scarpulla, unpubl.) promoters, which rely on functional NRF-1 and NRF-2 recognition sites for their activities.

A subset of respiratory genes does not appear to be regulated by NRF-1 or NRF-2. Other well-characterized regulatory factors have been implicated in the expression of these genes. The transcription factor $\mathrm{Sp} 1$ is associated with the activation and/or repression of cytochrome $c_{1}$ (Li et al. 1996b) and adenine nucleotide translocase 2 genes (Li et al. 1996a), both of which lack NRF sites (Zaid et al. 1999). Sp1 sites are also common to many GC-rich promoters including those that are NRF-dependent. The muscle-specific COX subunits, COXVIaH and COXVIII, are also lacking NRF sites but depend on MEF-2 and/or E-box consensus elements for their expression (Wan and Moreadith 1995). Thus, the same or similar factors required for the expression of other muscle-specific genes are linked to the regulation of these tissue-specific $C O X$ subunits. In contrast, the promoter of the ubiquitously expressed liver isoform, COXVIaL, depends on NRF-1 and NRF-2 as well as Sp1 for full activity (Seelan et al. 1996). This is consistent with the observation that in gene pairs encoding ubiquitous and tissue-specific isoforms of a given protein, the NRF-1 site, when present, is associated with the ubiquitously expressed gene (Virbasius et al. 1993a). Finally, the initiator element transcription factor YY1 participates in the expression of certain $C O X$ genes. Functional YY1-binding sites have been detected in the promoters of genes encoding $C O X \mathrm{Vb}$ (Basu et al. 1997) and COXVIIc (Seelan and Grossman 1997). Multiple YY1 sites in the COXVb promoter bind YY1 and possibly other factors, and at least one of these sites helps confer a negative regulatory effect on $C O X V b$ promoter activity (Basu et al. 1997). In the COXVIIc promoter, two YY1 sites in conjunction with an NRF-2 site act as positive regulators of promoter activity (Seelan and Grossman 1997). It is also important to note that regulation of most nuclear genes encoding mitochondrial enzymes upstream of the respiratory chain is NRF-1/NRF-2-independent. For example, genes encoding mitochondrial fatty acid oxidation enzymes are regulated by the peroxisome proliferator-activated receptor alpha (PPAR $\alpha$ ) and other NRF-1-independent regulatory pathways (Gulick et al. 1994). Thus, any unifying transcriptional model of mitochondrial biogenesis needs to account for the expression of genes that are NRF-independent.

There are several reports suggesting that nuclear and mitochondrial genes are controlled by common cis-acting elements that are the targets of the same or similar transcription factors. Sequence similarities to the OXBOX/REBOX (Haraguchi et al. 1994) and Mt (Suzuki et al. 1995) elements have been localized to the mitochondrial D-loop. The ability of these elements and their nuclear gene counterparts to bind proteins from crude extracts with the same specificity has been taken as evidence for shared regulatory factors between the two genetic systems (Haraguchi et al. 1994). Similarly, other nuclear factors, such as thyroid hormone receptors, have been implicated in mitochondrial gene expression (for review, see Wrutniak-Cabello et al. 2001). However, there is no evidence that these proteins can use the mitochondrial transcriptional machinery to direct mitochondrial gene expression.

\section{The critical role of transcriptional coactivators in the mitochondrial biogenic regulatory cascade: The PPARy coactivator-1 (PGC-1) family}

As described above, the mitochondrial biogenic program involves the integration of multiple transcriptional regulatory pathways controlling the expression of both nuclear and mitochondrial genes. This highlights a mechanistic enigma fundamental to the control of mitochondrial biogenesis. How is the activity of multiple transcription factors (e.g., NRF-1, NRF-2, PPAR $\alpha$, mtTFA) coordinately regulated during the mitochondrial biogenic process? Moreover, in the context of such complex integration, how is cell- and tissue-specific function achieved? For example, mitochondria within the brown adipocyte are poised for uncoupled mitochondrial respiration, whereas in other tissues such as heart, mitochondrial respiration is largely coupled for high-level ATP production. To add to the complexity, skeletal muscle is capable of supporting both coupled and uncoupled respiration. New insight into this problem was provided by the discovery of the transcriptional coactivator PPAR $\gamma$ coactivator $1 \alpha$ (PGC$1 \alpha)$ by Spiegelman and colleagues (Puigserver et al. 1998). $P G C-1 \alpha$ was cloned in a yeast two-hybrid screen for brown adipose-specific factors that interacted with the adipogenic nuclear receptor PPAR $\gamma$ (Puigserver et al. 1998). PGC- $1 \alpha$ serves as a direct transcriptional coacti- 
vator of PPAR $y$ and is a member of a growing list of proteins that coactivate transcription factors through direct protein-protein interactions (for review, see Knutti and Kralli 2001; Puigserver and Spiegelman 2003).

Transcriptional coactivators serve multiple functions including modification of chromatin through posttranslational histone acetylation, direct interaction with the RNA polymerase II complex, mRNA processing, and recruitment of other transcriptional coactivators (for review, see Robyr et al. 2000; Belandia and Parker 2003). Present evidence indicates that PGC- $1 \alpha$ coactivates its targets via recruitment of additional coactivators with histone acetylase activity, such as SRC-1 (Puigserver et al. 1999). In addition, the PGC- $1 \alpha$ molecule contains domains capable of interacting with and processing premRNA (Monsalve et al. 2000). PGC- $1 \alpha$ also interacts directly with the TRAP/Mediator complex (Wallberg et al. 2003). Unlike most known transcriptional coactivators, PGC- $1 \alpha$ is unique in that it exhibits a tissue-enriched expression pattern and is highly inducible (Puigserver et al. 1998; Knutti and Kralli 2001; Puigserver and Spiegelman 2003). PGC- $1 \alpha$ is enriched in brown adipose, heart, slow-twitch skeletal muscle, and kidney-tissues with high-capacity mitochondrial systems. The expression of the $P G C-1 \alpha$ gene is rapidly induced by cold exposure, short-term exercise, and fasting; physiologic conditions known to increase the demand on mitochondria to produce heat or ATP (Puigserver et al. 1998; Wu et al. 1999; Goto et al. 2000; Lehman et al. 2000; Baar et al. 2002; Terada et al. 2002; Irrcher et al. 2003; Pilegaard et al. 2003; Terada and Tabata 2003). These latter observations suggested that PGC- $1 \alpha$ is involved in the physiologic control of mitochondrial function.

Several lines of evidence indicate that the transcriptional coactivator PGC- $1 \alpha$ serves as a key regulator of mitochondrial biogenesis in mammals. First, studies focused on the biologic function of PGC- $1 \alpha$ revealed that it activates the transcription of mitochondrial uncoupling protein-1 (UCP-1) through interactions with the nuclear hormone receptors PPAR $\gamma$ and thyroid hormone receptor (Puigserver et al. 1998). These findings further supported a role for PGC-1 $\alpha$ in the process of mitochondrial uncoupled respiration and thermogenesis in brown adipose tissue. Second, forced expression studies in adipogenic and myogenic mammalian cell lines demonstrated that PGC-1 $\alpha$ markedly induces the expression of NRF-1, NRF-2, and Tfam (Wu et al. 1999). PGC-1 $\alpha$ can also interact directly with and coactivate NRF-1 on the Tfam gene promoter. Third, studies in primary cardiac myocytes in culture and in the hearts of transgenic mice have demonstrated that overexpression of $P G C-1 \alpha$ up-regulates the expression of genes involved in mitochondrial fatty acid oxidation, most of which are PPAR $\alpha$ targets, in addition to NRF-1 targets (Lehman et al. 2000). Cardiacspecific overexpression of $P G C-1 \alpha$ in transgenic mice leads to massive mitochondrial proliferation, ultimately resulting in cardiomyopathy and death (Lehman et al. 2000). Interestingly, in neonatal cardiac myocytes in culture, PGC- $1 \alpha$ induces mitochondria that support largely coupled respiration consistent with the known ATP-gen- erating function of this organelle in heart (Lehman et al. 2000). Lastly, forced expression of $P G C-1 \alpha$ in skeletal muscle of transgenic mice triggers mitochondrial proliferation and the formation of mitochondrial-rich type I, oxidative ("slow-twitch") muscle fibers (Lin et al. 2002b). Collectively, these results indicate that PGC- $1 \alpha$ is capable of promoting mitochondrial biogenesis through its coactivating effects on key factors such as NRF-1.

The gain-of-function studies described above provide compelling evidence that PGC- $1 \alpha$ serves as a transcriptional coactivator to promote mitochondrial biogenesis in postnatal mammalian tissues. Although $N R F-1$ is a key target of PGC-1 $\alpha$, it is clear that this transcription factor does not control all of the components of the mitochondrial biogenic response. Multiple PGC- $1 \alpha$ targets have now been identified, indicating that this coactivator serves as a pleiotropic regulator of multiple pathways involved in cellular energy metabolism within and outside of the mitochondrion (Knutti and Kralli 2001; Puigserver and Spiegelman 2003). Following the identification of PPAR $\gamma$ as the initial PGC- $1 \alpha$ transcription factor target, a variety of additional members of the nuclear receptor superfamily have been shown to interact with PGC-1 $\alpha$. This list includes PPAR $\alpha$ (Vega et al. 2000), thyroid hormone receptor (Puigserver et al. 1998), retinoid receptors (Puigserver et al. 1998), glucocorticoid receptor (Knutti et al. 2000), estrogen receptor (Puigserver et al. 1998; Knutti et al. 2000; Tcherepanova et al. 2000), HNF-4 (Rhee et al. 2003), and estrogen-related receptors (ERRs; Huss et al. 2002; Schreiber et al. 2003). In addition, several non-nuclear-receptor PGC-1 $\alpha$ partners have been identified, in addition to NRF-1, including myocyte-enhancing factor-2 (MEF-2; Michael et al. 2001) and FOX-01 (Puigserver et al. 2003). Although several of the PGC- $1 \alpha$ partners serve functions outside of the mitochondrion such as HNF-4 and FOX-01 (gluconeogenesis; Rhee et al. 2003; Puigserver et al. 2003) and MEF-2 (glucose transport; Michael et al. 2001), others are linked to the mitochondrial biogenic transcriptional regulatory program. For example, PGC- $1 \alpha$ coactivates the nuclear receptor $\operatorname{PPAR} \alpha$, a key regulator of nuclear genes encoding mitochondrial fatty acid oxidation enzymes /Vega et al. 2000). More recently, PGC-1 $\alpha$ was found to coactivate the orphan nuclear receptors $\mathrm{ERR} \alpha$ and ERR $\gamma$ (Huss et al. 2002; Schreiber et al. 2003). Although the exact biologic function of ERRs has not been delineated, $\mathrm{ERR} \alpha$ and ERR $\gamma$ are enriched in tissues with high mitochondrial oxidative capacity including brown adipose tissue and heart. In addition, medium-chain acyl-CoA dehydrogenase $(M C A D)$, a known $\operatorname{PPAR} \alpha$ target that catalyzes the initial step in mitochondrial fatty acid $\beta$-oxidation, is also regulated by $\operatorname{ERR} \alpha$ (Sladek et al. 1997; Vega and Kelly 1997; Huss et al. 2002). These results suggest that $\mathrm{ERR} \alpha$ and $\operatorname{PPAR} \alpha$ may drive distinct but overlapping mitochondrial pathways downstream of PGC- $1 \alpha$.

PGC- $1 \alpha$ is now known to be a member of a family of transcriptional coactivators. The first PGC- $1 \alpha$ relative, PGC-1-related coactivator (PRC), was identified through a database search (Andersson and Scarpulla 2001). PRC 
contains several domains that are homologous to PGC$1 \alpha$ including an acidic N-terminal region, an LXXLL motif for interacting with nuclear receptors, a proline-rich region, and regions known to interact with RNA (Fig. 2). Although overall homology between PGC-1 $\alpha$ and PRC is relatively low, the similarity of domains suggests related function. In contrast to $P G C-1 \alpha, P R C$ is largely ubiquitously expressed, is only slightly induced in response to cold exposure, and is cell-cycle-regulated (Andersson and Scarpulla 2001). However, functional studies indicate that PRC may be capable of regulating mitochondrial function in a manner similar to PGC- $1 \alpha$. PRC interacts directly with and coactivates NRF-1 via natural NRF-1 recognition sites in the 5-ALAS gene promoter (Andersson and Scarpulla 2001). Additional experiments have revealed that PRC activates the transcription of another known NRF-1 target, cytochrome c, but requires the cooperation of other factors including CREB (Andersson and Scarpulla 2001). A third member of the family, PGC$1 \beta$ (also termed PGC-1-related estrogen receptor coactivator or PERC), was also identified through database searching (Kressler et al. 2002; Lin et al. 2002a). PGC-1 $\beta$ exhibits a greater degree of homology to PGC-1 $\alpha$ than PRC (Fig. 2). The expression pattern of PGC-1 $\beta$ exhibits similarities with that of PGC- $1 \alpha$ such as enrichment in heart and brown adipose tissue. Furthermore, $P G C-1 \beta$ is induced by fasting but not in response to cold exposure (Lin et al. 2002a). PGC-1 $\beta$ interacts with HNF- $4 \alpha$, NRF1, and ERR $\alpha$ (Kressler et al. 2002; Lin et al. 2002a; Kamei et al. 2003). PGC-1 $\beta$ also interacts with Host Cell Factor $(\mathrm{HCF})$, a cellular protein implicated in cell cycle regulation (Lin et al. 2002a). The relevance of this latter interaction to the regulation of mitochondrial function is unknown.

The differences in regulation and tissue-expression patterns of PGC-1 family members suggest that each confers distinct biologic responses. In support of this idea, recent work by the Spiegelman laboratory has provided evidence that PGC- $1 \alpha$ and PGC-1 $\beta$ isoforms exert coactivator-specific bioenergetic effects (St-Pierre et al. 2003). Specifically, overexpression studies in $\mathrm{C}_{2} \mathrm{C}_{12}$ myotubes demonstrated that although both PGC- $1 \alpha$ and
PGC-1 $\beta$ increase mitochondrial proton leak rates, cells expressing $P G C-1 \alpha$ have a higher proportion of mitochondrial respiration linked to proton leak. PGC-1 $\beta$ was shown to preferentially induce the expression of genes involved in the removal of reactive oxygen species that themselves could serve as activators of uncoupling (St-Pierre et al. 2003).

\section{Upstream signaling events involved in the control of mitochondrial biogenesis: PGC-1 as an integrative coactivator}

The observation that $P G C-1 \alpha$ gene expression is rapidly induced by cold exposure in the brown adipose tissue of mice spawned a series of observational studies aimed at determining whether additional physiologic stimuli are capable of modulating the expression of this coactivator. It was subsequently shown that $P G C-1 \alpha$ gene expression is induced by exercise in rodent and human skeletal muscle (Goto et al. 2000; Baar et al. 2002; Terada et al. 2002; Terada and Tabata 2003; Pilegaard et al. 2003) and by short-term starvation in the heart and liver of mice (Lehman et al. 2000; Rhee et al. 2003). The transcriptional regulatory mechanisms involved in the regulation of $P G C-1 \alpha$ gene expression in response to physiologic stimuli are only beginning to be understood. In one example, the transcription factor CREB can promote hepatic gluconeogenesis, in part through its induction of $P G C-1 \alpha$ via direct binding to a functional CRE in the $P G C-1 \alpha$ promoter (Herzig et al. 2001). More recently, $P G C-1 \alpha$ transcription was shown to be regulated by members of the MEF2 transcription factor family and repressed by class II histone deacetylases (HDACs; Czubryt et al. 2003).

Signal transduction pathways play a major role in the physiologic regulation of mitochondrial function and biogenesis; therefore, it is not surprising that $P G C-1 \alpha$ activity and expression are regulated by similar signaling pathways. In tissues poised for mitochondrial thermogenesis, such as brown adipose, the $\beta$-adrenergic/cAMP pathway is upstream of the PGC- $1 \alpha$-mediated regulation of targets such as UCP-1 (Puigserver et al. 1998). A sig-

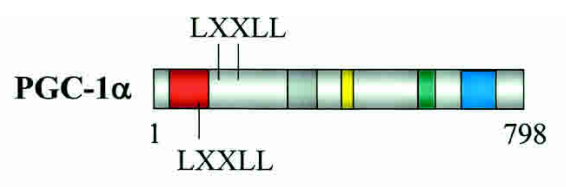

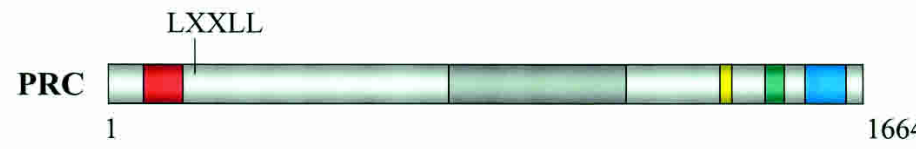

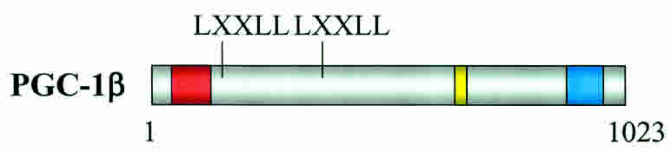

- activation $\square$ - HCF binding $\square-$ RS domain
1664

Figure 2. The PGC-1 family of coactivators. Schematic representation of the primary structures of PGC- $1 \alpha$, PRC, and PGC1ß. The leucine-rich (LXXLL) domains critical for interaction with nuclear receptors are also shown. Additional specific shared domains are indicated as denoted by the key at the bottom. 
nificant body of evidence focused largely on skeletal muscle indicates that in response to contractile activity, calcium-dependent signaling pathways trigger a cascade of regulatory events leading to increased formation of oxidative fiber types and a marked increase in mitochondrial number and function (Holloszy and Coyle 1984; Chin et al. 1998). Several important gain-of-function studies have now provided evidence for regulatory links between calcineurin A, calcium/calmodulin-dependent protein kinase (CaMK), PGC- $1 \alpha$, and skeletal muscle mitochondrial biogenesis. First, overexpression of $\mathrm{CaMK}$ in the skeletal muscle of transgenic mice triggers a robust mitochondrial biogenesis associated with an induction of PGC-1 $\alpha$ expression (Wu et al. 2002). Second, overexpression of $P G C-1 \alpha$ in the skeletal muscle of transgenic mice leads to the formation of slow-twitch skeletal muscle fibers and an induction of genes involved in mitochondrial oxidative metabolism (Lin et al. 2002b). Third, studies performed in myogenic cell lines indicate that both calcineurin A and CaMK are capable of activating $P G C-1 \alpha$ gene transcription (Handschin et al. 2003). The calcineurin A-mediated activation of $P G C-1 \alpha$ transcription is dependent on MEF2 response elements, whereas CaMK-mediated regulation requires CREB-binding sites.

Several other signal transduction pathways have been implicated in the control of $P G C$-1 expression and activity. p38 MAPK activates $P G C-1 \alpha$ by releasing repression of an unidentified factor and by increasing PGC- $1 \alpha$ protein stability (Knutti et al. 2001; Puigserver et al. 2001). p38 MAPK can also activate the PGC-1 $\alpha$ partner, $P P A R \alpha$, suggesting that activation of this signaling pathway influences mitochondrial fatty acid oxidation (Barger et al. 2001). However, the role of the p38 MAPK pathway in regulating mitochondrial biogenesis is not known. More recently, evidence has emerged that nitric oxide (NO) activates mitochondrial biogenesis in a variety of cell types including adipocytes, and HeLa cells (Nisoli et al. 2003). The mitochondrial thermogenic response is significantly altered in mice lacking eNOS. This NO effect is dependent on cGMP and linked to $P G C-1 \alpha$ activation. These results raise the intriguing possibility that mitochondrial biogenesis is one of the important effects of NO activation. Given the known role of NO as a vasodilator, it is tempting to speculate that this key upstream regulatory pathway coordinately regulates downstream events including an increase in the capacity to use oxygen in mitochondria.

\section{Summary}

Over the past decade, significant new insight has been gained into the circuitry of molecular regulatory cascades controlling mitochondrial biogenesis and function (Fig. 3). The interdependence of nuclear and mitochondrial genomes has evolved with the emergence of the mitochondrion as a eukaryotic organelle. It is likely that the complexity of the mammalian organism mandates a complex regulatory network that provides for the dynamic coordinate control of nuclear and mitochondrial genes during development and in the adult. This regulatory circuitry not only triggers mitochondrial biogenesis in response to developmental and physiologic cues, but also confers cell- and tissue-specific features. New insight into the dynamic control of mitochondrial function and biogenesis has been provided by the identification of relevant transcription factors, transcriptional coactivators, and upstream signaling events. However, the mechanisms involved in the control of cell-specific mitochondrial phenotypes and the full cast of transcriptional regulatory factors comprise an exciting investigative frontier. New experimental approaches such as the
Figure 3. PGC-1 serves a central integrative role in the transcriptional regulatory cascade upstream of the mitochondrial biogenic response. A schematic representation of the mitochondrial biogenic regulatory cascade, including known PGC-1 partners and putative upstream signaling pathways.

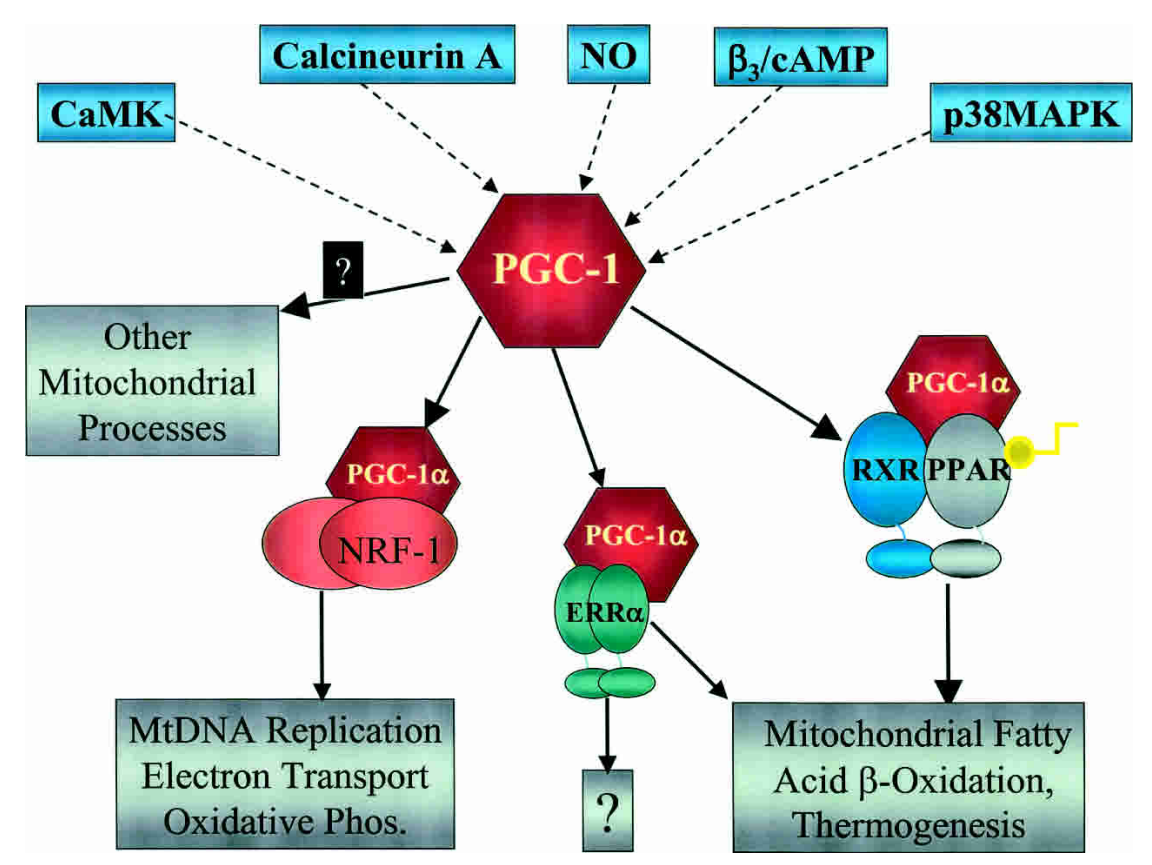


delineation of tissue-specific mitochondrial proteomes (Mootha et al. 2003) should provide an excellent framework for future studies aimed at understanding the molecular events involved in defining the mitochondrial phenotype.

\section{Acknowledgments}

Special thanks to Mary Wingate for assistance with manuscript preparation and Janice Huss for critical review of the manuscript. Work in the authors' laboratories is supported by United States Public Health Service Grants DK45416, HL58493, HL57278, HL61006 to D.P.K. and GM32525 to R.C.S. from the National Institutes of Health.

\section{References}

Andersson, U. and Scarpulla, R.C. 2001. PGC-1-Related coactivator, a novel, serum-inducible coactivator of nuclear respiratory factor-1-dependent transcription in mammalian cells. Mol. Cell. Biol. 21: 3738-3749.

Antoshechkin, I. and Bogenhagen, D.F. 1995. Distinct roles for two purified factors in transcription of Xenopus mitochondrial DNA. Mol. Cell. Biol. 15: 7032-7042.

$\mathrm{Au}$, H.C. and Scheffler, I.E. 1998. Promoter analysis of the human succinate dehydrogenase iron-protein gene. Both nuclear respiratory factors NRF-1 and NRF-2 are required. Eur. J. Biochem. 251: 164-174.

Baar, K., Wende, A.R., Jones, T.E., Marison, M., Nolte, L.A., Chen, M., Kelly, D.P., and Holloszy, J.O. 2002. Adaptations of skeletal muscle to exercise: Rapid increase in the transcriptional coactivator PGC-1 $\alpha$. FASEB J. 16: 1879-1886.

Barger, P.M., Browning, A.C., Garner, A.N., and Kelly, D.P. 2001. p38 MAP kinase activates PPAR $\alpha$ : A potential role in the cardiac metabolic stress response. J. Biol. Chem. 276: 44495-44501.

Basu, A., Lenka, N., Mullick, J., and Avadhani, N.G. 1997. Regulation of murine cytochrome oxidase $\mathrm{Vb}$ gene expression in different tissues and during myogenesis-Role of a YY-1 factor-binding negative enhancer. I. Biol. Chem. 272: 58995908.

Batchelor, A.H., Piper, D.E., De la Brousse, F.C., McKnight, S.L., and Wolberger, C. 1998. The structure of GABP $\alpha / \beta$ : An ETS domain ankyrin repeat heterodimer bound to DNA. Science 279: 1037-1041.

Belandia, B. and Parker, M.G. 2003. Nuclear receptors: A rendezvous for chromatin remodeling factors. Cell 114: 277280.

Bergeron, R., Ren, J.M., Cadman, K.S., Moore, I.K., Perret, P., Pypaert, M., Young, L.H., Semenkovich, C.F., and Shulman, G.I. 2001. Chronic activation of AMP kinase results in NRF-1 activation and mitochondrial biogenesis. Am. I. Physiol. Endocrinol. Metabol. 281: E1340-E1346.

Blesa, J.R, Hernandez, J.M., and Hernandez-Yago, J. 2003. NRF-2 transcription factor is essential in promoting human Tomm70 gene expression. Mitochondrion (in press).

Bogenhagen, D.F. 1996. Interaction of mtTFB and mtRNA polymerase at core promoters for transcription of Xenopus laevis mtDNA. J. Biol. Chem. 271: 12036-12041.

Bogenhagen, D.F. and Clayton, D.A. 2003. The mitochondrial DNA replication bubble has not burst. Trends Biochem. Sci. 28: 357-360.

Brown, T.A. and Clayton, D.A. 2002. Release of replication termination controls mitochondrial DNA copy number after depletion with 2',3'-dideoxycytidine. Nucleic Acids Res. 30: 2004-2010.

Carter, R.S., Bhat, N.K., Basu, A., and Avadhani, N.G. 1992. The basal promoter elements of murine cytochrome $c$ oxidase subunit IV gene consist of tandemly duplicated ETS motifs that bind to GABP-related transcription factors. I. Biol. Chem. 267: 23418-23426.

Chau, C.A., Evans, M.J., and Scarpulla, R.C. 1992. Nuclear respiratory factor 1 activation sites in genes encoding the $\gamma$-subunit of ATP synthase, eukaryotic initiation factor $2 \alpha$, and tyrosine aminotransferase. Specific interaction of purified NRF-1 with multiple target genes. J. Biol. Chem. 267: 69997006.

Chin, E.R., Olson, E.N., Richardson, J.A., Yang, Q., Humphries, C., Shelton, J.M., Wu, H., Zhu, W., Bassel-Duby, R., and Williams, R.S. 1998. A calcineurin-dependent transcriptional pathway controls skeletal muscle fiber type. Genes \& Dev. 12: 2499-2509.

Chinenov, Y., Henzl, M., and Martin, M.E. 2000. The $\alpha$ and $\beta$ subunits of the GA-binding protein form a stable heterodimer in solution. J. Biol. Chem. 275: 7749-7756.

Choi, Y.S., Lee, H.K., and Pak, Y.K. 2002. Characterization of the $5^{\prime}$-flanking region of the rat gene for mitochondrial transcription factor A (Tfam). Biochim. Biophys. Acta Gene Struct. Expression 1574: 200-204.

Clayton, D.A. 2000. Transcription and replication of mitochondrial DNA. Hum. Reprod. 15: 11-17.

Czubryt, M.P., McAnally, J., Fishman, G.I., and Olson, E.N. 2003. Regulation of peroxisome proliferator-activated receptor $\gamma$ coactivator $1 \alpha$ (PGC- $1 \alpha$ ) and mitochondrial function by MEF2 and HDAC5. Proc. Nat1. Acad. Sci. 100: 1711-1716.

Dairaghi, D.J., Shadel, G.S., and Clayton, D.A. 1995. Addition of a 29 residue carboxyl-terminal tail converts a simple HMG box-containing protein into a transcriptional activator. J. Mol. Biol. 249: 11-28.

Diffley, J.F. and Stillman, B. 1991. A close relative of the nuclear, chromosomal high-mobility group protein HMG1 in yeast mitochondria. Proc. Natl. Acad. Sci. 88: 78647868.

Elbehti-Green, A., Au, H.C., Mascarello, J.T., Ream-Robinson, D., and Scheffler, I.E. 1998. Characterization of the human SDHC gene encoding one of the integral membrane proteins of succinate-quinone oxidoreductase in mitochondria. Gene 213: $133-140$.

Evans, M.J. and Scarpulla, R.C. 1988. Both upstream and intron sequence elements are required for elevated expression of the rat somatic cytochrome $c$ gene in COS-1 cells. Mol. Cell. Biol. 8: 35-41.

-1989. Interaction of nuclear factors with multiple sites in the somatic cytochrome $c$ promoter. Characterization of upstream NRF-1, ATF and intron Spl recognition sites. J. Biol. Chem. 264: 14361-14368.

Falkenberg, M., Gaspari, M., Rantanen, A., Trifunovic, A., Larsson, N.-G., and Gustafsson, C.M. 2002. Mitochondrial transcription factors $\mathrm{B} 1$ and $\mathrm{B} 2$ activate transcription of human mtDNA. Nat. Genet. 31: 289-294.

Fisher, R.P., Lisowsky, T., Parisi, M.A., and Clayton, D.A. 1992. DNA wrapping and bending by a mitochondrial high mobility group-like transcriptional activator protein. I. Biol. Chem. 267: 3358-3367.

Garesse, R. and Vallejo, C.G. 2001. Animal mitochondrial biogenesis and function: A regulatory cross-talk between two genomes. Gene 263: 1-16.

Goto, M., Terada, S., Kato, M., Katoh, M., Yokozeki, T., Tabata, I., and Shimokawa, T. 2000. cDNA cloning and mRNA analysis of PGC-1 in epitrochlearis muscle in swimming- 
exercised rats. Biochem. Biophys. Res. Commun. 274: 350354.

Gugneja, S. and Scarpulla, R.C. 1997. Serine phosphorylation within a concise amino-terminal domain in nuclear respiratory factor 1 enhances DNA binding. J. Biol. Chem. 272: 18732-18739.

Gugneja, S., Virbasius, J.V., and Scarpulla, R.C. 1995. Four structurally distinct, non-DNA-binding subunits of human nuclear respiratory factor 2 share a conserved transcriptional activation domain. Mol. Cell. Biol. 15: 102-111.

Gugneja, S., Virbasius, C.A., and Scarpulla, R.C. 1996. Nuclear respiratory factors 1 and 2 utilize similar glutamine-containing clusters of hydrophobic residues to activate transcription. Mol. Cell. Biol. 16: 5708-5716.

Gulick, T., Cresci, S., Caira, T., Moore, D.D., and Kelly, D.P. 1994. The peroxisome proliferator activated receptor regulates mitochondrial fatty acid oxidative enzyme gene expression. Proc. Natl. Acad. Sci. 91: 11012-11016.

Handschin, C., Rhee, J., Lin, J., Tam, P.T., and Spiegelman, B.M. 2003. An autoregulatory loop controls peroxisome proliferator-activated receptor $\gamma$ coactivator $1 \alpha$ expression in muscle. Proc. Natl. Acad. Sci. 100: 7111-7116.

Haraguchi, Y., Chung, A.B., Neill, S., and Wallace, D.C. 1994. OXBOX and REBOX, overlapping promoter elements of the mitochondrial $\mathrm{F}_{\text {OF1-ATP }}$ synthase $\beta$ subunit gene. OXBOX/ REBOX in the ATPsyn $\beta$ promoter. J. Biol. Chem. 269: 93309334.

Herzig, R.P., Scacco, S., and Scarpulla, R.C. 2000. Sequential serum-dependent activation of CREB and NRF-1 leads to enhanced mitochondrial respiration through the induction of cytochrome c. J. Biol. Chem. 275: 13134-13141.

Herzig, S., Long, F., Jhala, U.S., Hedrick, S., Quinn, R., Bauer, A., Rudolph, D., Schutz, G., Yoon, C., Puigserver, P., et al. 2001. $\mathrm{CREB}$ regulates hepatic gluconeogenesis through the coactivator PGC-1. Nature 413: 179-183.

Hirawake, H., Taniwaki, M., Tamura, A., Amino, H., Tomitsuka, E., and Kita, K. 1999. Characterization of the human SDHD gene encoding the small subunit of cytochrome $b$ $(c y b S)$ in mitochondrial succinate-ubiquinone oxidoreductase. Biochim. Biophys. Acta 1412: 295-300.

Holloszy, J.O. and Coyle, E.F. 1984. Adaptations of skeletal muscle to endurance exercise and their metabolic consequences. J. Applied Physiol. 56: 831-838.

Huo, L. and Scarpulla, R.C. 2001. Mitochondrial DNA instability and peri-implantation lethality associated with targeted disruption of nuclear respiratory factor 1 in mice. Mol. Cell. Biol. 21: 644-654.

Huss, J.M., Kopp, R.P., and Kelly, D.P. 2002. PGC- $1 \alpha$ coactivates the cardiac-enriched nuclear receptors estrogen-related receptor- $\alpha$ and - $\gamma$. J. Biol. Chem. 277: 40265-40274.

Irrcher, I., Adhihetty, P.J., Sheehan, T., Joseph, A.-M., and Hood, D.A. 2003. PPAR $\gamma$ coactivator- $1 \alpha$ expression during thyroid hormone- and contractile activity-induced mitochondrial adaptations. Am. I. Physiol. Cell Physiol. 284: C1669C1677.

Kamei, Y., Ohizumi, H., Fujitani, Y., Nemoto, T., Tanaka, T., Takahashi, N., Kawada, T., Miyoshi, M., Ezaki, O., and Kakizuka, A. 2003. PPAR $\gamma$ coactivator $1 \beta /$ ERR ligand 1 is an ERR protein ligand, whose expression induces a high-energy expenditure and antagonizes obesity. Proc. Natl. Acad. Sci. 100: $12378-12383$.

Knutti, D. and Kralli, A. 2001. PGC-1, a versatile coactivator. Trends Endocrinol. Metab. 12: 360-365.

Knutti, D., Kaul, A., and Kralli, A. 2000. A tissue-specific coactivator of steroid receptors. Mol. Cell. Biol. 20: 24112422 .
Knutti, D., Kressler, D., and Kralli, A. 2001. Regulation of the transcriptional coactivator PGC-1 via MAPK-sensitive interaction with a corepressor. Proc. Natl. Acad. Sci. 98: 97139718.

Kressler, D., Schreiber, S.N., Knutti, D., and Kralli, A. 2002. The PGC-1-related protein PERC is a selective coactivator of estrogen receptor $\alpha$. J. Biol. Chem. 277: 13918-13925.

LaMarco, K.L. and McKnight, S.L. 1989. Purification of a set of cellular polypeptides that bind to the purine-rich cis-regulatory element of herpes simplex virus immediate early genes. Genes \& Dev. 3: 1372-1383.

Larsson, N.-G., Oldfors, A., Holme, E., and Clayton, D.A. 1994. Low levels of mitochondrial transcription factor A in mitochondrial DNA depletion. Biochem. Biophys. Res. Commun. 200: 1374-1381.

Larsson, N.-G., Wang, J.M., Wilhelmsson, H., Oldfors, A., Rustin, P., Lewandoski, M., Barsh, G.S., and Clayton, D.A. 1998. Mitochondrial transcription factor $\mathrm{A}$ is necessary for mtDNA maintenance and embryogenesis in mice. Nat. Genet. 18: 231-236.

Lehman, J.J., Barger, P.M., Kovacs, A., Saffitz, J.E., Medeiros, D., and Kelly, D.P. 2000. PPAR $\gamma$ coactivator-1 (PGC-1) promotes cardiac mitochondrial biogenesis. J. Clin. Invest. 106: $847-856$.

Li, R., Hodny, Z., Luciakova, K., Barath, P., and Nelson, B.D. 1996a. Spl activates and inhibits transcription from separate elements in the proximal promoter of the human adenine nucleotide translocase 2 (ANT2) gene. J. Biol. Chem. 271: 18925-18930.

Li, R., Luciakova, K., and Nelson, B.D. 1996b. Expression of the human cytochrome $c_{1}$ gene is controlled through multiple Spl-binding sites and an initiator region. Eur. J. Biochem. 241: 649-656.

Lin, J., Puigserver, P., Donovan, J., Tarr, P., and Spiegelman, B.M. 2002a. Peroxisome proliferator-activated receptor $\gamma$ coactivator $1 \beta$ (PGC-1 $\beta$ ), a novel PGC-1-related transcription coactivator associated with host cell factor. J. Biol. Chem. 277: 1645-1648.

Lin, J., Wu, H., Tarr, P.T., Zhang, C.Y., Wu, Z., Boss, O., Michael, L.F., Puigserver, P., Isotanni, E., Olson, E.N., et al. 2002b. Transcriptional co-activator PGC-1 $\alpha$ drives the formation of slow-twitch muscle fibres. Nature 418: 797801 .

McCulloch, V. and Shadel, G.S. 2003. Human mitochondrial transcription factor $\mathrm{B} 1$ interacts with the $\mathrm{C}$-terminal activation region of h-mtTFA and stimulates transcription independently of its RNA methyltransferase activity. Mol. Cell. Biol. 23: 5816-5824.

McCulloch, V., Seidel-Rogol, B.L., and Shadel, G.S. 2002. A human mitochondrial transcription factor is related to RNA adenine methyltransferases and binds $S$-adenosylmethionine. Mol. Cell. Biol. 22: 1116-1125.

Michael, L.F., Wu, Z., Cheatham, R.B., Puigserver, P., Adelmant, G., Lehman, J.J., Kelly, D.P., and Spiegelman, B.M. 2001. Restoration of insulin-sensitive glucose transporter (GLUT4) gene expression in muscle cells by the transcriptional coactivator PGC-1. Proc. Natl. Acad. Sci. 98:38203825.

Miranda, S., Foncea, R., Guerreo, J., and Leighton, F. 1999. Oxidative stress and upregulation of mitochondrial biogenesis genes in mitochondrial DNA-depleted HeLa cells. Biochem. Biophys. Res. Commun. 258: 44-49.

Monsalve, M., Wu, Z., Adelmant, G., Puigserver, P., Fan, M., and Spiegelman, B.M. 2000. Direct coupling of transcription and mRNA processing through the thermogenic coactivator PGC-1. Mol. Cell 6: 307-316. 
Mootha, V.K., Bunkenborg, J., Olsen, J.V., Hjerrid, M., Wisniewski, J.R., Stahl, E., Bolouri, M.S., Ray, H.N., Sihag, S., Kamal, M., et al. 2003. Integrated analysis of protein composition, tissue diversity, and gene regulation in mouse mitochondria. Cell 115: 629-640.

Murakami, T., Shimomura, Y., Yosimura, A., Sokabe, M., and Fujitsuka, N. 1998. Induction of nuclear respiratory factor-1 expression by an acute bout of exercise in rat muscle. Biochem. Biophys. Acta 1381: 113-122.

Nisoli, E., Clementi, E., Paolucci, C., Cozzi, V., Tonello, C., Sciorati, C., Bracale, R., Valerio, A., Francolini, M., Moncada, S., et al. 2003. Mitochondrial biogenesis in mammals: The role of endogenous nitric oxide. Science 299: 896899.

Ojuka, E.O., Jones, T.E., Han, D.H., Chen, M., and Holloszy, J.O. 2003. Raising $\mathrm{Ca}^{2+}$ in L6 myotubes mimics effects of exercise on mitochondrial biogenesis in muscle. FASEB $I$. 17: 675-681.

Parisi, M.A., Xu, B., and Clayton, D.A. 1993. A human mitochondrial transcriptional activator can functionally replace a yeast mitochondrial HMG-box protein both in vivo and in vitro. Mol. Cell. Biol. 13: 1951-1961.

Pilegaard, H., Saltin, B., and Neufer, P.D. 2003. Exercise induces transient transcriptional activation of the PGC- $1 \alpha$ gene in human skeletal muscle. J. Physiol. 546: 851-858.

Poulton, J., Morten, K., Freeman-Emmerson, C., Potter, C., Sewry, C., Dubowitz, V., Kidd, H., Stephenson, J., Whitehouse, W., Hansen, F.J., et al. 1994. Deficiency of the human mitochondrial transcription factor h-mtTFA in infantile mitochondrial myopathy is associated with mtDNA depletion. Hum. Mol. Genet. 3: 1763-1769.

Poyton, R.O. and McEwen, J.E. 1996. Crosstalk between nuclear and mitochondrial genomes. Annu. Rev. Biochem. 65: 563607.

Puigserver, P. and Spiegelman, B.M. 2003. Peroxisome proliferator-activated receptor- $\gamma$ coactivator $1 \alpha(\mathrm{PGC}-1 \alpha)$ : Transcriptional coactivator and metabolic regulator. Endocrine Rev. 24: 78-90.

Puigserver, P., Wu, Z., Park, C.W., Graves, R., Wright, M., and Spiegelman, B.M. 1998. A cold-inducible coactivator of nuclear receptors linked to adaptive thermogenesis. Cell 92: 829-839.

Puigserver, P., Adelmant, G., Wu, Z., Fan, M., Xu, J., O'Malley, B., and Spiegelman, B.M. 1999. Activation of PPAR $\gamma$ coactivator-1 through transcription factor docking. Science 286: 1368-1371.

Puigserver, P., Rhee, J., Lin, J., Wu, Z., Yoon, J.C., Zhang, C.-Y., Kraquss, S., Mootha, V.K., Lowell, B.B., and Spiegelman, B.M. 2001. Cytokine stimulation of energy expenditure through p38 MAP kinase activation of PPAR $\gamma$ coactivator-1. Mol. Cell 8: 971-982.

Puigserver, P., Rhee, J., Donovan, J., Walkey, C.J., Yoon, J.C., Oriente, F., Kitamura, Y., Altomonte, J., Dong, H., Accili, D., et al. 2003. Insulin-regulated hepatic gluconeogenesis through FOXO1-PGC-1 $\alpha$ interaction. Nature 423: 550555.

Rantanen, A., Jansson, M., Oldfors, A., and Larsson, N.-G. 2001. Downregulation of Tfam and mtDNA copy number during mammalian spermatogenesis. Mamm. Genome 12: 787792.

Rantanen, A., Gaspari, M., Falkenberg, M., Gustafssn, C.M., and Larsson, N.-G. 2003. Characterization of the mouse genes for mitochondrial transcription factors B1 and B2. Mamm. Genome 14: 1-6.

Rhee, J., Inoue, Y., Yoon, J.C., Puigserver, P., Fam, M., Gonzalez, F.J., and Spiegelman, B.M. 2003. Regulation of hepatic fast- ing response by PPAR $\gamma$ coactivator- $1 \alpha$ (PGC-1 $1 \alpha$ ): Requirement for hepatocyte nuclear factor $4 \alpha$ in gluconeogenesis. Proc. Natl. Acad. Sci. 100: 4012-4017.

Robyr, D., Wolffe, A.P., and Wahli, W. 2000. Nuclear hormone receptor coregulators in action: Diversity for shared tasks. Mol. Endocrinol. 14: 329-347.

Scarpulla, R.C. 1997. Nuclear control of respiratory chain expression in mammalian cells. J. Bioenerg. Biomembr. 29: 109-119.

- 1999. Nuclear transcription factors in cytochrome $c$ and cytochrome oxidase expression. In Frontiers of cellular bioenergetics: Molecular biology, biochemistry, and physiopathology (ed. S. Papa et al.), pp. 553-591. Plenum, London.

- 2002. Nuclear activators and coactivators in mammalian mitochondrial biogenesis. Biochim. Biophys. Acta 1576: $1-14$.

Schreiber, S.N., Knutti, D., Brogli, K., Uhlmann, T., and Kralli, A. 2003. The transcriptional coactivator PGC-1 regulates the expression and activity of the orphan nuclear receptor estrogen-related receptor $\alpha(\mathrm{ERR} \alpha)$. J. Biol. Chem. 278: 90139018.

Seelan, R.S. and Grossman, L.I. 1997. Structural organization and promoter analysis of the bovine cytochrome $c$ oxidase subunit VIIc gene-A functional role for YY1. J. Biol. Chem. 272: $10175-10181$.

Seelan, R.S., Gopalakrishnan, L., Scarpulla, R.C., and Grossman, L.I. 1996. Cytochrome $c$ oxidase subunit VIIa liver isoform: Characterization and identification of promoter elements in the bovine gene. J. Biol. Chem. 271:21122120.

Shadel, G.S. and Clayton, D.A. 1997. Mitochondrial DNA maintenance in vertebrates. Annu. Rev. Biochem. 66: 409435.

Sladek, R., Bader, J.-A., and Giguere, V. 1997. The orphan nuclear receptor estrogen-related receptor $\alpha$ is a transcriptional regulator of the human medium-chain acyl coenzyme a dehydrogenase gene. Mol. Cell. Biol. 17: 5400-5409.

St-Pierre, J., Lin, J., Krauss, S., Tarr, P.T., Yang, R., Newgard, C.B., and Spiegelman, B.M. 2003. Bioenergetic analysis of peroxisome proliferator-activated receptor $\gamma$ coactivators $1 \alpha$ and $1 \beta$ (PGC- $1 \alpha$ and PGC-1 $\beta$ ) in muscle cells. J. Biol. Chem. 278: $26597-26603$.

Suliman, H.B., Carraway, M.S., Welty-Wolf, K.E., Whorton, A.R., and Piantados, C.A. 2003. Lipopolysaccharide stimulates mitochondrial biogenesis via activation of nuclear respiratory factor-1. J. Biol. Chem. 278: 41510-41518.

Suzuki, H., Suzuki, S., Kumar, S., and Ozawa, T. 1995. Human nuclear and mitochondrial Mt element-binding proteins to regulatory regions of the nuclear respiratory genes and to the mitochondrial promoter region. Biochem. Biophys. Res. Commun. 213: 204-210.

Takahashi, Y., Kako, K., Arai, H., Ohishi, T., Inada, Y. Takehara, A., Fukamizu, A., and Munekata, E. 2002. Characterization and identification of promoter elements in the mouse COX17 gene. Biochim. Biophys. Acta 1574: 359364.

Tcherepanova, I., Puigserver, P., Norris, J.D., Speigelman, B.M., and McDonnell, D.P. 2000. Modulation of estrogen receptor- $\alpha$ transcriptional activity by coactivator PGC-1. I. Biol. Chem. 275: 16302-16308.

Terada, S. and Tabata, I. 2003. Effects of acute bouts of running and swimming exercise on PGC- $1 \alpha$ protein expression in rat epitrochlearis and soleus muscle. Am. J. Physiol. Endocrinol. Metabol. 286: E208-E216.

Terada, S., Goto, M., Kato, M., Kawanaka, K., Shimokawa, T., and Tabata, I. 2002. Effects of low-intensity prolonged exer- 
cise on PGC-1 mRNA expression in rat epitrochlearis muscle. Biochem. Biophys. Res. Commun. 296: 350-354.

Thompson, C.C., Brown, T.A., and McKnight, S.L. 1991. Convergence of ETS- and notch-related structural motifs in a heteromeric DNA binding complex. Science 253: 762-768.

Tiranti, V., Savoia, A., Forti, F., D'Apolito, M.F., Centra, M., Racchi, M., and Zeviani, M. 1997. Identification of the gene encoding the human mitochondrial RNA polymerase (hmtRPOL) by cyberscreening of the expressed sequence tags database. Hum. Mol. Genet. 6: 615-625.

Vega, R.B. and Kelly, D.P. 1997. A role for estrogen-related receptor $\alpha$ in the control of mitochondrial fatty acid $\beta$-oxidation during brown adipocyte differentiation. J. Biol. Chem. 272: 31693-31699.

Vega, R.B., Huss, J.M., and Kelly, D.P. 2000. The coactivator PGC-1 cooperates with peroxisome proliferator-activated receptor $\alpha$ in transcriptional control of nuclear genes encoding mitochondrial fatty acid oxidation enzymes. Mol. Cell. Biol. 20: $1868-1876$.

Virbasius, J.V. and Scarpulla, R.C. 1991. Transcriptional activation through ETS domain binding sites in the cytochrome $c$ oxidase subunit IV gene. Mol. Cell. Biol. 11: 5631-5638.

-1994. Activation of the human mitochondrial transcription factor A gene by nuclear respiratory factors: A potential regulatory link between nuclear and mitochondrial gene expression in organelle biogenesis. Proc. Natl. Acad. Sci. 91: 1309-1313.

Virbasius, C.A., Virbasius, J.V., and Scarpulla, R.C. 1993a. NRF-1, an activator involved in nuclear-mitochondrial interactions, utilizes a new DNA-binding domain conserved in a family of developmental regulators. Genes \& Dev. 7: 24312445.

Virbasius, J.V., Virbasius, C.A., and Scarpulla, R.C. 1993b. Identity of GABP with NRF-2, a multisubunit activator of cytochrome oxidase expression, reveals a cellular role for an ETS domain activator of viral promoters. Genes \& Dev. 7: 380392.

Wallberg, A.E., Yamamura, S., Malik, S., Spiegelman, B.M., and Roeder, R.G. 2003. Coordination of p300-mediated chromatin remodeling and TRAP/mediatro function through coactivator PGC-1 $\alpha$. Mol. Cell 12: 1137-1149.

Wan, B. and Moreadith, R.W. 1995. Structural characterization and regulatory element analysis of the heart isoform of cytochrome $c$ oxidase VIa. J. Biol. Chem. 270: 26433-26440.

Wrutniak-Cabello, C., Casas, F., and Cabello, G. 2001. Thyroid hormone action in mitochondria. J. Mol. Endocrinol. 26: 6777.

Wu, Z., Puigserver, P., Andersson, U., Zhang, C., Adelmant, G., Mootha, V., Troy, A., Cinti, S., Lowell, B., Scarpulla, R.C., et al. 1999. Mechanisms controlling mitochondrial biogenesis and respiration through the thermogenic coactivator PGC-1. Cell 98: 115-124.

Wu, H., Kanatous, S.B., Thurmond, F.A., Gallardo, T., Isotani, E., Bassel-Duby, R., and Williams, R.S. 2002. Regulation of mitochondrial biogenesis in skeletal muscle by CaMK. Science 296: 349-352.

Zaid, A., Li, R., Luciakova, K., Barath, P., Nery, S., and Nelson, B.D. 1999. On the role of the general transcription factor Sp1 in the activation and repression of diverse mammalian oxidative phosphorylation genes. J. Bioenerg. Biomembr. 31: 129-135.

Zitomer, R.S. and Lowry, C.V. 1992. Regulation of gene expression by oxygen in Saccharomyces cerevisiae. Microbiol. Rev. 56: 1-11. 


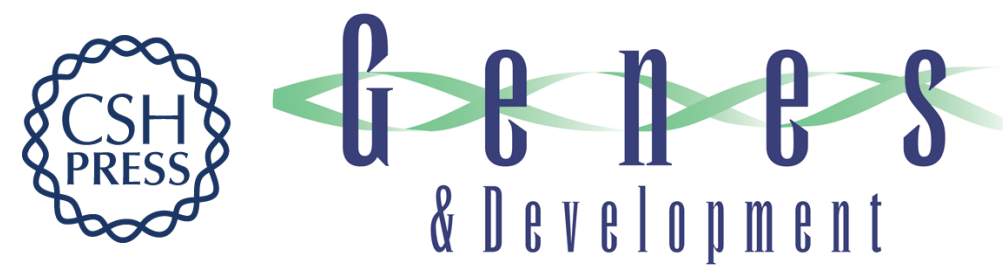

\title{
Transcriptional regulatory circuits controlling mitochondrial biogenesis and function
}

\author{
Daniel P. Kelly and Richard C. Scarpulla
}

Genes Dev. 2004, 18:

Access the most recent version at doi:10.1101/gad.1177604

$\begin{array}{ll}\text { References } & \begin{array}{l}\text { This article cites } 103 \text { articles, } 54 \text { of which can be accessed free at: } \\ \text { http://genesdev.cshlp.org/content/18/4/357.full.html\#ref-list-1 }\end{array}\end{array}$

License

Email Alerting Receive free email alerts when new articles cite this article - sign up in the box at the top Service right corner of the article or click here.

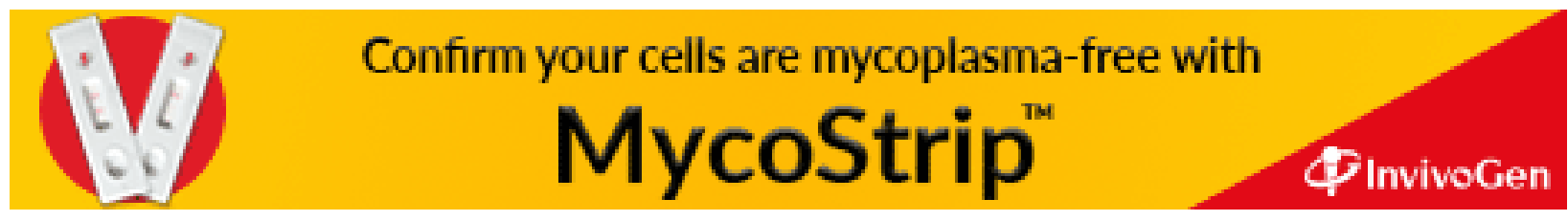

